\title{
Uncommon association of anomalous circumflex coronary and aortic coarctation
}

\author{
Susan C. Pumacayo-Cárdenas* and Regina de la Mora-Cervantes \\ Department of Cardiovascular Computed Tomography, Instituto Nacional de Cardiología Ignacio Chávez, Mexico City, Mexico
}

A 25-year-old patient, with a history of coartectomy and aortic arch extension at 2 years of age and stent aortoplasty at age 13 due to recoarctation. He was asymptomatic at rest and during effort and denied episodes of chest pain, dyspnea, and syncope. Clinical examination, electrocardiogram, and echocardiography were normal. A control computed tomography angiography for the coarctation of the aorta, which was obtained using a non-synchronized acquisition protocol, showed a normally looking, permeable stent, with no evidence of recoarctation. As an incidental finding (Fig. 1), anomalous origin of the circumflex coronary artery (CX) from the left pulmonary artery was detected, with caudal course between both pulmonary arteries, where it gave rise to the sinus node branch and then ran medial to the left atrium to head toward the left atrioventricular groove. It is important noting that the CX flow is retrograde, given the hyperdensity observed from the CX to the pulmonary artery. In the volumetric reconstruction (Fig. 2A), the stent was observed under the left subclavian artery and the CX course. It is important to note the collateral coronary circulation, of the anterior descending and right coronary artery, through its diagonal and marginal acute branches, respectively (Fig. 2 B and C).

Anomalous origin of the $\mathrm{CX}$ from the pulmonary artery (ACCAPA) is a congenital coronary anomaly with low prevalence $(<0.3 \%)^{1-2}$, and it is less common when it originates in the left pulmonary artery ${ }^{2}$. Clinical presentation is highly variable, and being a very rare pathology, it will depend on collateral coronary circulation; findings ranging from sudden death and myocardial infarction to asymptomatic cases have been reported. Association with other congenital heart diseases occurs in $3-36 \%$ of cases, with the most commonly described including those that involve embryological formation of the cone trunk, such as aortopulmonary window, tetralogy of Fallot, ductus arteriosus, pulmonary stenosis and coarctation of the aorta, and, more rarely, Scimitar syndrome ${ }^{2-3}$.

This case is important because it presents an infrequent pathology (ACCAPA) and even more so because it originates in the left pulmonary branch, associated with aortic coarctation, in the context of an asymptomatic patient whose diagnosis was established by a follow-up tomography that was not aimed at the coronary arteries, which also showed collateral circulation of the right and anterior descending coronary arteries, which allowed patient survival.

\section{Funding}

The present investigation has not received specific aid from public or commercial sector agencies or non-profit entities.

\section{Correspondence:}

*Susan C. Pumacayo-Cárdenas

Col. Belisario Dominguez, Sec. XVI, Del. Tlalpan

C.P. 14080 , Ciudad de Mexico, Mexico

E-mail: pumacayocardenas@gmail.com
Available online: $30-10-2019$ Arch Cardiol Mex (Eng). 2019;89(2):169-170 www.archivoscardiologia.com 2604-7063 @ 2019 Instituto Nacional de Cardiología Ignacio Chávez. Published by Permanyer México SA de CV. This is an open access article under the CC BY-NC-ND license (http://creativecommons.org/licenses/by-nc-nd/4.0/). 

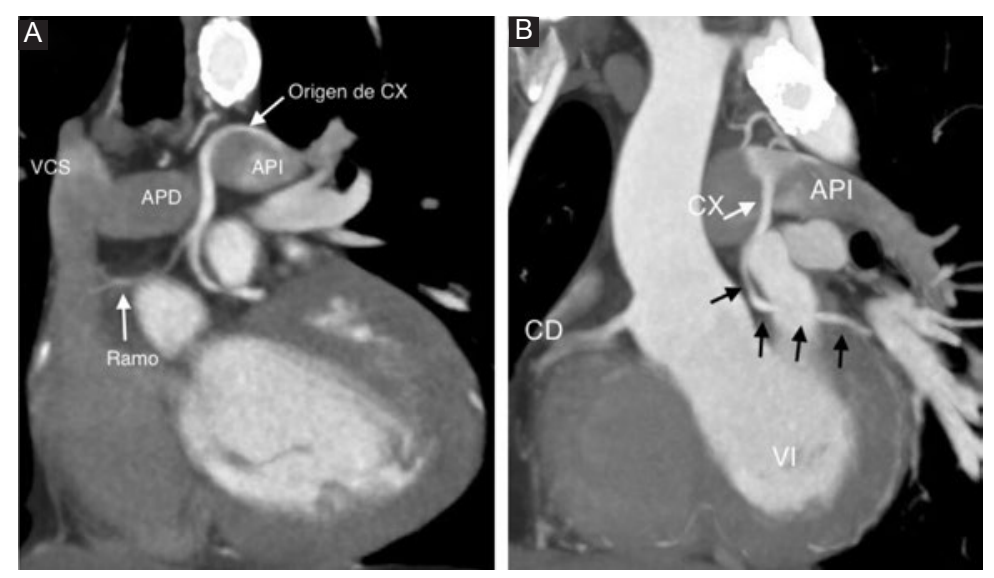

Figure 1. CT angiography, FLASH protocol. A: origin of the circumflex coronary artery (CX) from the LPA proximal portion and internal face, with caudal course between LPA and RPA, where it gives rise to the sinus node branch; B: CX origin and retrograde flow toward LPA (white arrow) are observed. CX course toward the left atrioventricular groove (black arrows). Origen de CX: CX origin; API: left pulmonary artery; APD: right pulmonary artery; VCS: superior vena cava; Ramo: branch; CD: right coronary artery; VI: left ventricle.

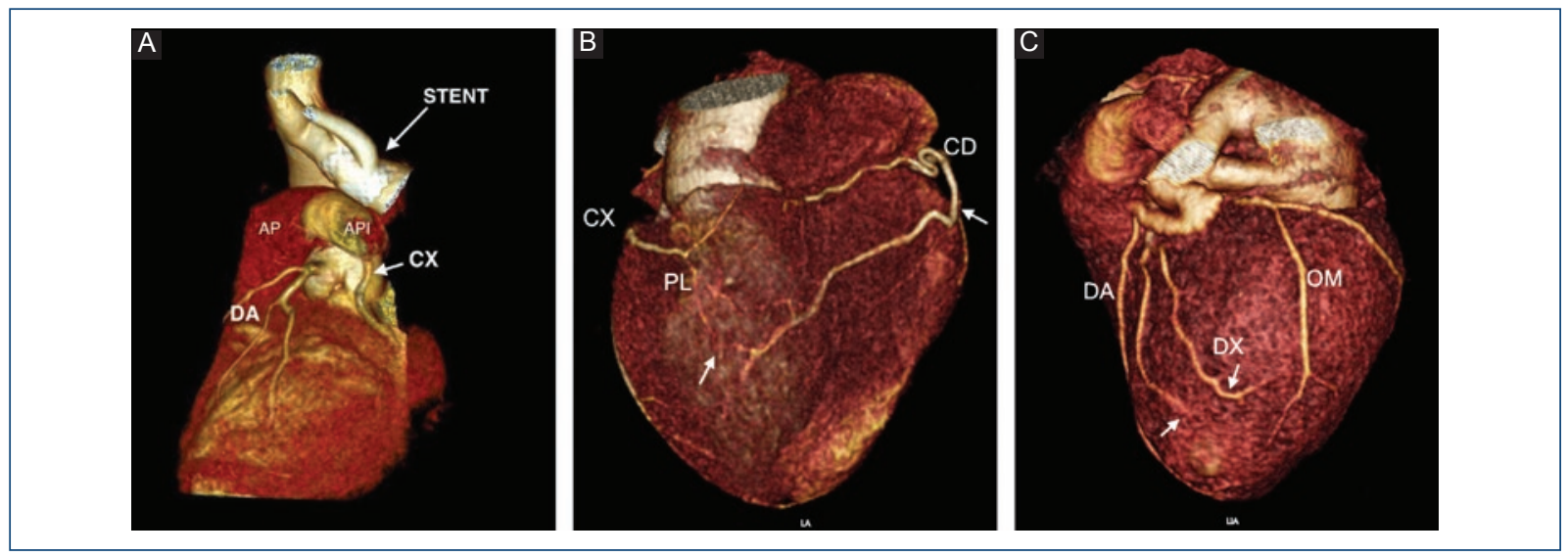

Figure 2. Volumetric reconstruction. A: left lateral view. The stent is observed under the left subclavian artery. Note the CX origin and relationship with the AD artery with preserved origin and trajectory and its first diagonal with good caliber and development; B: inferior face view showing acute marginal branch with good development and caliber, providing circulation toward the PL territory; C: left lateral view showing first and second diagonal branches with good development, which provide collateral circulation toward the OM branch territory. AP: pulmonary artery trunk; API: left pulmonary artery; CD: right coronary artery; CX: circumflex coronary artery; DA: anterior descending; DX: diagonal branches 1 and 2; PL: CX posterolateral branch; MO: marginal oblique.

\section{Conflicts of interest}

The authors declare that they have no conflicts of interest.

\section{Ethical disclosures}

Protection of people and animals. The authors declare that no experiments were carried out on humans or animals for this research.

Confidentiality of data. The authors declare that they have followed the protocols of their work center on the publication of patient data.
Right to privacy and informed consent. The authors declare that no patient data appear in this article.

\section{References}

1. Çitaku H, Kamberi L, Gorani D, Koçinaj D, Krasniqi X. Anomalous origin of left circumflex artery. Med Arch. 2015;69:423-4.

2. Sekelyk R, Mykychak Y, Fedevych O, Yemets I. Anomalous origin of circumflex coronary artery from right pulmonary artery associated with coarctation of the aorta: a case report of surgical treatment. World $\mathrm{J}$ Pediatr Congenit Heart Surg. 2011;5:97-9.

3. Aktas D, Erdem A, Çelik N, Kamalı H, Sarıtas T. A rare coronary anomaly with masked diagnosis: Anomalous left circumflex artery from right pulmonary artery. Turk Kardiyol Dern Ars. 2015;43(6):551-3. 